\title{
Efectos de la sustitución parcial de maíz por harina integral de mandioca sobre variables productivas de cerdos en crecimiento
}

\author{
Pochon, D.O. ${ }^{1}$; Koslowski, H.A. ${ }^{1-2}$; Picot, J.A. ${ }^{1}$; Navamuel, J.M. ${ }^{1}$ \\ ${ }^{1}$ Cátedra de Bioestadística, ${ }^{2}$ Cátedra de Nutrición y Alimentación Animal, Facultad de Ciencias Veterinarias, \\ UNNE, Sargento Cabral 2139, Corrientes (3400), Argentina. Tel. 03783-425753 (interno 138). \\ E-mail: dopoch@vet.unne.edu.ar.
}

\begin{abstract}
Resumen
Pochon, D.O.; Koslowski, H.A.; Picot, J.A.; Navamuel, J.M.: Efectos de la sustitución parcial de maiz por harina integral de mandioca sobre variables productivas de cerdos en crecimiento. Rev. vet. 21: 1, 38-42, 2010. La importancia de utilizar mandioca en la dieta del cerdo radica en su alto contenido de carbohidratos, siendo una excelente fuente de energía metabolizable. Esta energía es usualmente aportada por el maíz y constituye alrededor del 60 al 80\% de una ración. No obstante, existen forrajes regionales potencialmente capaces de reemplazar a los tradicionalmente usados en la alimentación porcina, cuya eficiencia debe ser previamente evaluada. El trabajo tuvo la finalidad de valorar el efecto de dietas a base de mandioca sobre las variables productivas de cerdos en crecimiento, reemplazando el maíz por distintos niveles de sustitución con raíz de mandioca. Se utilizaron cuatro cerdos machos enteros, con pesos promedios de 40,4 $\pm 0,5 \mathrm{~kg}$, alojados en corrales individuales de 2 $\mathrm{m}^{2}$, durante 7 días de adaptación a las dietas y 14 de mediciones. Los diferentes niveles de sustitución de maíz fueron: $0,20,40$ y $60 \%$ de raíz de mandioca para las dietas 1, 2, 3 y 4 respectivamente, todas isoenergéticas e isoproteicas. Las variables bajo estudio recibieron análisis descriptivo y su respuesta se estimó por ANOVA con un diseño en cuadrado latino y un alfa de 5\%. No se registraron diferencias significativas entre las cuatro dietas para las variables productivas evaluadas. La dieta 2 fue la que generó mayor ganancia total y ganancia diaria de peso $\left(12 \pm 1,63 \mathrm{~kg}\right.$ y $1,08 \pm 0,13 \mathrm{~kg}$.día $\left.{ }^{-1}\right)$, así como el más alto consumo diario de alimento $\left(2,82 \pm 0,32 \mathrm{~kg} \cdot \mathrm{MS}\right.$.día $\left.{ }^{-1}\right)$. Este cerdo requirió $2,64 \pm 0,38 \mathrm{~kg}$ de alimento por $\mathrm{kg}$ de peso vivo ganado. La menor ganancia de peso se registró en el cerdo alimentado con la dieta $3(10,75 \pm 1,26 \mathrm{~kg})$, reflejada en una ganancia diaria de $0,97 \pm 0,11 \mathrm{~kg}$.día ${ }^{-1}$. Se concluye que bajo las presentes condiciones de trabajo, el buen desempeño productivo del grupo experimental permite avalar la factibilidad del reemplazo parcial del maíz por harina de raíz de mandioca, en las raciones para cerdos en crecimiento.
\end{abstract}

Palabras clave: cerdo, alimentos alternativos, mandioca, ganancia de peso.

\begin{abstract}
Pochon, D.O.; Koslowski, H.A.; Picot, J.A.; Navamuel, J.M.: Effects on productive variables with partial substitution of corn with manioc in the diet of pigs during growing stage. Red. Vet. 21:1, 38-42, 2010. The use of manioc for pig diets is important because of its high composition of carbohydrates, being and excellent source of metabolizable energy. The latter is traditionally achieved by supplying corn as $60-80 \%$ of the ration. Agroecological alternatives for a particular geographical area have to be taken into account when considering the replacement of traditional feeding systems for pigs. The purpose of this work was to determine the effects of manioc-based diets on productive variables by replacing traditional corn-based diets with different proportions of integral manioc flour in growing pigs. For this purpose, four male pigs weighing $40.4 \pm 0.5 \mathrm{~kg}$ were located in $2 \mathrm{~m}^{2}$ corrals with food and water ad libitum during 7 days for adaptation and 14 days for the assay. Different percentages of corn substitution were: balanced ration plus $0,20,40$ and $60 \%$ of manioc flour, for diets $1,2,3$ and 4, respectively. Proportions of manioc were isoenergetic and isoproteic for all the cases. Variables under analysis were submitted to descriptive analysis and response was estimated by ANOVA with a latin square design, being alpha of $5 \%$. No significant differences for the studied variables were observed for the four diets. Diet 2 showed the highest total and daily weight gain $\left(12 \pm 1.63 \mathrm{~kg}\right.$ and $1.08 \pm 0.13 \mathrm{~kg}$ day $\left.^{-1}\right)$ as well as the highest daily food intake
\end{abstract}


$\left(2.82 \pm 0.32 \mathrm{~kg} . M S\right.$.day $\left.{ }^{-1}\right)$. This animal needed $2.64 \pm 0.38 \mathrm{~kg}$ of food per $\mathrm{kg}$ of live weight gain. Least live weight gain was registered in the animal fed with diet $3(10.75 \pm 1.26 \mathrm{~kg})$, indicating a daily live weight gain of $0.97 \pm 0.11 \mathrm{~kg}^{-d a y}{ }^{-1}$. It can be concluded that, under this assay conditions, the favorable performance of the experimental group allows the feasibility for the partial replacement of corn with manioc flour for diets of pigs during the growing stage.

Key words: pig, alternative food, manioc, weight gain.

\section{INTRODUCCIÓN}

Tradicionalmente la producción porcina se realiza en las mismas zonas donde se obtiene su principal fuente de alimentación. Argentina no escapa a esta premisa: el gran volumen de la producción de cerdos coincide con las zonas de cultivos de maíz y soja, ambos de uso masivo en la preparación de sus alimentos. Estos cultivos también son empleados en la alimentación humana, lo que determina una importante competencia e influye marcadamente en la fluctuación de los precios de dichos productos ${ }^{29}$.

Existen ciertas situaciones propias de cada país, que ocasionan que en un momento determinado la disponibilidad de las principales fuentes de energía (maíz y sorgo) sea limitada. Tal restricción puede deberse a insuficiente producción nacional, escasez de divisas para la importación, o alto costo por competir con la alimentación humana. Esta situación crea la necesidad de buscar alternativas que resulten nutricionalmente aceptables, y que puedan solventar el problema en un momento de crisis, así como producir adecuados rendimientos productivos ${ }^{21,22}$.

El manejo económico de las producciones pecuarias depende en un alto porcentaje de los costos de alimentación, los cuales están determinados por el uso de recursos forrajeros disponibles en la región. Para lograr que esta elección sea viable, incluso para grandes productores de cerdos, se deben implantar políticas agrarias que incentiven la producción local de materias primas no tradicionales en alimentación animal, como mandioca, batata, algunas leguminosas, caña de azúcar y otras.

El uso de materias primas regionales, no tradicionales en la alimentación animal, permite la reducción de los costos de producción con el consiguiente ahorro de divisas, promueven el desarrollo de nuevas industrias basadas en la transformación de productos industriales en alimentos para animales y, finalmente, determinan una disminución en el precio de los insumos ${ }^{6}$.

Ciertos recursos forrajeros alternativos presentan limitaciones nutricionales en sus contenidos de energía y fibra, aminoácidos limitantes, existencia de sustancias tóxicas (factores antinutricionales) y otras. Tales dificultades pueden afectar los rendimientos productivos, causando una disminución en la eficiencia de conversión de alimentos y un incremento en el costo económico para producir una unidad de producto. No obstante, en situaciones de emergencia y mediante un balance adecuado de nutrientes y un nivel bajo de utili- zación, estos productos pueden sustituir adecuadamente las fuentes de energía tradicionales en los alimentos balanceados para cerdos ${ }^{1,3,5}$.

La información sobre el valor nutritivo de los alimentos alternativos es muy escasa en nuestra zona; para incluirlos en las dietas es necesario caracterizar sus propiedades nutritivas, que incluyen no sólo su composición química, sino también las características físicas que posibilitan determinar su calidad teniendo en cuenta cómo influyen en los procesos digestivos y fisiológicos del animal ${ }^{30,31}$.

La mandioca, Manihot esculenta Cranz, pertenece a la familia Euphorbiaceae, es de ciclo estival y originaria de América, recibiendo numerosos apelativos identificatorios, como "yuca", "cassava " y " tapioca" en Centroamérica, "mandioca" en Argentina y Brasil, "mandió" en Paraguay. Otros nombres empleados son "suahili", "mhogo", "omowgo" 12 . El cultivo de mandioca presenta bajos requerimientos edafológicos y se produce adecuadamente en suelos de escasa fertilidad como los llanos ${ }^{23}$, condiciones que la hacen ventajosa como una alternativa para la producción animal. La mandioca y el maíz fueron las primeras plantas cultivadas en el continente americano y los principales recursos alimenticios de los pueblos originarios. $\mathrm{La}$ mandioca, fue uno de los primeros cultivos tropicales americanos que el hombre industrializó como fuente de almidón.

Argentina produce 200.000 tn de raíces frescas con un rendimiento medio de $10-15 \mathrm{tn} / \mathrm{ha}$ de raíces frescas y $2,5 \mathrm{tn} /$ ha de almidón ${ }^{13}$. Esto representa 10 millones de $\mathrm{kcal} / \mathrm{ha} / \mathrm{año}$ de energía metabolizable, en comparación con los 7,3 millones que produce el maíz y superior al de cualquier otro cereal adaptado al trópico ${ }^{9}$.

Su composición nutricional depende de la variedad, suelo, fertilización y condiciones ambientales. La raíz de la mandioca contiene $65 \%$ de agua y $35 \%$ de materia seca. La harina contiene normalmente de 86 a $90 \%$ de materia seca ${ }^{9}$. La pulpa de la raíz contiene más materia seca $(37,8 \%)$ que la cáscara $(27,8 \%)$ y representa el $86,8 \%$ de la raíz; el resto (13,2\%), está constituido por la cáscara ${ }^{28}$. El principal componente de la materia seca de la harina de yuca son los carbohidratos, donde un 64 a 72\% corresponde a almidones, de los cuales el $20 \%$ es amilosa y $69 \%$ es amilopectina, existiendo pequeñas cantidades de sacarosa, maniqueosa y fructosa ${ }^{17}$. El alto contenido de carbohidratos hace de la harina de mandioca una excelente fuente de energía. Numerosos trabajos han reportado el contenido de energía digestible de este producto. La mayoría de los inves- 
Tabla 1. Composición centesimal de las dietas ensayadas (\%).

\begin{tabular}{lcccc}
\hline dieta & 1 & 2 & 3 & 4 \\
\hline maíz & 65 & 52 & 39 & 23 \\
mandioca & 0 & 13 & 23 & 37 \\
soja & 30 & 30 & 33 & 35 \\
minerales* $^{*}$ & 5 & 5 & 5 & 5 \\
\hline
\end{tabular}

${ }^{*}$ Complemix Cerdos 5\% ${ }^{\mathrm{NR}}$.

Tabla 2. Análisis proximal de las dietas ( $\%$ en base fresca).

\begin{tabular}{lcccc}
\hline dieta & 1 & 2 & 3 & 4 \\
\hline proteína bruta & 20 & 18 & 18 & 19 \\
extracto etéreo & 2,8 & 3,2 & 2,2 & 3,0 \\
fibra bruta & 3,8 & 3,9 & 4,0 & 4,6 \\
cenizas totales & 5,6 & 4,4 & 5,8 & 5,2 \\
\hline
\end{tabular}

tigadores reportan valores que fluctúan entre $3.500 \mathrm{a}$ $4.185 \mathrm{kcal} / \mathrm{kg}^{10,28}$, y otros hasta $4.310 \mathrm{kcal} / \mathrm{kg}^{20}$.

La variación en el contenido de energía dependerá de la calidad de la harina y de la proporción de cáscara (criterios de elaboración). Bajo condiciones prácticas de producción porcícola un valor promedio para formulación de raciones de $3.800 \mathrm{kcal} / \mathrm{kg}$ (90\% materia seca) produjo adecuados rendimientos productivos ${ }^{9}$. La mayoría de las variedades de mandioca contiene bajos niveles de proteína, con valores que no exceden al 3\% (promedios del 2,3\%) ${ }^{28}$; sin embargo, el ICA en Colombia ha desarrollado variedades (Manihot carthagenesis) con niveles de hasta $15,4 \%$ de proteína en base seca ${ }^{16}$. Los niveles de calcio y fósforo son del $0,12 \mathrm{y}$ $0,16 \%$, respectivamente.

El uso de la harina de mandioca en la alimentación animal no es una propuesta nueva para los países centroamericanos, sin embargo los niveles de inclusión en las dietas para cerdos aún no han sido claramente determinados, pues diferentes pruebas exploratorias sugieren que tiene limitantes en su inclusión, debido a factores antinutricionales y al manejo en la preparación por la polvosidad del producto ${ }^{1,2}$. Uno del los factores de mayor importancia que ha restringido el uso masivo de raíces de mandioca en cerdos es la presencia de los glucósidos cianogénicos linamarina y lotaustralina $(\mathrm{HCN})$, como así también la presencia de taninos ${ }^{24}$. Los glucósidos, al tomar contacto con la enzima linamarasa o por hidrólisis ácida, liberan ácido cianhídrico; poderoso inhibidor de la respiración celular. La reacción se inicia cuando las raíces se aplastan (daño celular) o durante su almacenamiento, procesamiento o manipulación ${ }^{7}$.

Los fundamentos señalados precedentemente, nos condujeron a la búsqueda de fuentes alternativas de energía de producción local, con el propósito de sustituir al máximo posible el porcentaje de inclusión de maíz con expectativas de disminuir costos de producción y dependencia del mismo ${ }^{27}$.

El objetivo del trabajo fue determinar ganancia de peso, consumo de alimento y tasa de conversión en cerdos en crecimiento alimentados con una dieta cuya fuente de energía estuvo constituida por distintos niveles de harina integral de raíz de mandioca sustituyendo a los granos de maíz.

\section{MATERIAL Y MÉTODOS}

Los cerdos utilizados en el ensayo $(n=4)$ procedían de la Escuela Regional de Agricultura Ganadería e Industrias Afines (Corrientes, Argentina) y eran cruzas mejoradas de similar fenotipo, sexo macho (enteros), clínicamente sanos y desparasitados. Las unidades experimentales registraron un peso inicial promedio de $40,4 \pm 0,5 \mathrm{~kg}$ y permanecieron alojados en corrales individuales de $2 \mathrm{~m}^{2}$ provistos de bebederos automáticos (agua ad libitum) y comederos.

Luego de ser cosechada, la raíz de mandioca fue lavada, triturada y secada al sol. La deshidratación constituye un paso necesario para la conservación de la calidad de la materia prima, así como para la eliminación o disminución de los principios tóxicos, amén de facilitar la preparación de alimentos balanceados. Para lograr una buena deshidratación, las raíces fueron molidas en fragmentos pequeños ${ }^{35}$. Además del tamaño de los fragmentos, también es importante considerar la forma de los mismos, habida cuenta que el vegetal posee una estructura celular en la cual las fibras están dispuestas en dirección axial. No tiene el mismo efecto reducir la longitud del trozo de raíz, que reducir su diámetro ${ }^{32}$. Para satisfacer las necesidades de los cerdos, al momento de formular las dietas cada preparación se adaptó a las recomendaciones del Nutrient Requirements of Swine ${ }^{25}$.

El ensayo incluyó 4 dietas (Tabla 1) que se correspondieron con los diferentes niveles de sustitución de harina de mandioca. Las dietas 1, 2, 3 y 4 incluyeron mandioca en porcentajes del $0,20,40$ y $60 \%$, sustituyendo las respectivas proporciones de maíz molido. Todas las dietas fueron equivalentes en energía y proteínas (Tabla 2).

Se evaluó diferencia de peso inicial-final $(\mathrm{kg})$, ganancia diaria de peso $\left(\mathrm{kg}^{-}\right.$día $\left.^{-1}\right)$, consumo diario de alimento (g.MS ${ }^{-1}$.día) y conversión alimenticia $(\mathrm{kg})$, con un diseño en cuadrado latino con 4 repeticiones (una por dieta), en 4 potreros, durante 7 días de adaptación y 14 días de medición (efecto fila), luego se rotaron al azar los tratamientos con el objeto que cada animal (efecto columna) recibiera una ración diferente por 21 días en cada período.

Las variables analizadas fueron clasificadas como cuantitativas en escala continua. Con el objeto de realizar un análisis preliminar de los resultados, se aplicó estadística descriptiva, análisis de gráficos de cajas y patillas y de distribución. La respuesta se evaluó por análisis de la variancia con un diseño en cuadrado latino, mediante el siguiente modelo lineal aditivo: $\mathrm{Y}_{\mathrm{ijk}}=$ $\mu+\mathrm{K}_{\mathrm{k}}+f_{\mathrm{i}}+\mathrm{C}_{\mathrm{j}}+\mathrm{e}_{\mathrm{ijk}}{ }^{34}$. Todas las variables en estudio cumplieron con los supuestos de normalidad, homogeneidad de la variancia e independencia. 


\section{RESULTADOS Y DISCUSIÓN}

Las Tablas 3 a 6 describen las variables diferencia de peso, ganancia diaria de peso, consumo diario de alimento y conversión alimenticia obtenidas para cada dieta. El tratamiento que produjo mayor diferencia de peso $(12 \pm 1,63 \mathrm{~kg})$ fue la dieta 2 (20\% mandioca), en coincidencia con una mayor ganancia diaria de peso $\left(1,08 \pm 0,13 \mathrm{~kg}\right.$.día $\left.{ }^{-1}\right)$ y mayor consumo diario de alimento $\left(2,82 \pm 0,32 \mathrm{~kg} . M S\right.$. día $\left.^{-1}\right)$. Los animales asignados a esta dieta necesitaron menor cantidad de alimento $(2,64 \pm 0,38 \mathrm{~kg})$ por kilogramos de peso vivo ganado (conversión alimenticia). En ningún caso estas variables fueron significativamente diferentes al resto. En experiencias anteriores, aplicando la prueba de cafetería $^{27}$, la dieta 2 fue la segunda más visitada $(27 \%$ del total), indicando que hubo buena aceptación, selección y consumo diario.

Los cerdos que recibieron la dieta 3 (40\% de mandioca) fueron los que menos ganancia de peso alcanzaron, aunque las diferencias no fueron estadísticamente significativas. Consecuentemente, la ganancia diaria de peso fue baja, aunque el consumo diario de alimento y la conversión alimenticia fueron similares a las otras dietas (no significativas). Estas observaciones no coinciden con el número de visitas que recibió el comedero con esta ración, ya que fue la más concurrida $(66,3 \%$ de la visitas) ${ }^{27}$.

Los resultados aquí registrados son coincidentes con los obtenidos por otros investigadores al sustituir parcialmente el maíz por harina de mandioca ${ }^{14,15,18} \mathrm{y}$ harina de batata ${ }^{4,8,11}$, aunque en ninguno de los casos referenciados se observaron diferencias significativas.

Al evaluar raciones con diferentes niveles de harina de follaje de mandioca como fuente proteica en cerdos de engorde $(0,5,15$ y $25 \%)$, no se hallaron diferencias entre las variables ganancia diaria de peso, consumo diario de alimento y características de la canal ${ }^{26}$. En la formulación de raciones para cerdos, el aporte energético de $3.800 \mathrm{kcal} / \mathrm{kg}(90 \%$ de materia seca) produjo adecuados rendimientos productivos, concluyéndose que la harina de batata puede reemplazar al maíz como fuente energética en raciones para el engorde, siempre que sea suplementada con una fuente proteica ${ }^{5,9,10}$.

No se encontraron diferencias significativas con respecto a ganancia diaria de peso, consumo diario de alimento y conversión alimenticia, tanto en machos como en hembras, al comparar una dieta testigo con otra en la cual se reemplazó totalmente el maíz amarillo por harina de mandioca, sin presentarse mortalidad en ninguno de los tratamientos realizados ${ }^{33}$.

El consumo voluntario es probablemente el factor más importante desde el punto de vista pecuario, ya que los demás parámetros como la ganancia de peso y la conversión de alimento, dependerán en forma directa del consumo voluntario. En el comportamiento alimentario también gravitan considerablemente el medio interno del animal, los factores gastrointestinales, hormonales y metabólicos ${ }^{19}$.
Tabla 3. Diferencia de peso $(\mathrm{kg})$ para las distintas dietas.

\begin{tabular}{lcccc}
\hline dieta & $\overline{\mathrm{x}}(\mathrm{n}=4)$ & $\mathrm{DE}$ & $\mathrm{CV}(\%)$ & rango \\
\hline 1 & 11,25 & 1,50 & 13,0 & $10-13$ \\
2 & 12,00 & 1,63 & 13,6 & $10-14$ \\
3 & 10,75 & 1,26 & 9,0 & $9-12$ \\
4 & 11,25 & 0,50 & 5,0 & $11-12$ \\
\hline
\end{tabular}

$\overline{\mathrm{X}}$ promedio, DE: desvío estándar, CV: coeficiente de variación. Las diferencias entre promedios fueron no significativas.

Tabla 4. Ganancia diaria de peso $\left(\mathrm{kg} \cdot \mathrm{día}^{-1}\right)$ para las distintas dietas.

\begin{tabular}{lcccc}
\hline dieta & $\overline{\mathrm{x}}(\mathrm{n}=4)$ & $\mathrm{DE}$ & $\mathrm{CV}(\%)$ & rango \\
\hline 1 & 1,02 & 0,13 & 13,1 & $0,91-1,18$ \\
2 & 1,08 & 0,13 & 12,2 & $0,91-1,23$ \\
3 & 0,97 & 0,11 & 10,9 & $0,82-1,07$ \\
4 & 1,03 & 0,04 & 4,43 & $1,00-1,09$ \\
\hline
\end{tabular}

$\overline{\mathrm{X}}$ promedio, DE: desvío estándar, CV: coeficiente de variación. Las diferencias entre promedios fueron no significativas.

Tabla 5. Consumo diario de alimento (Kg.MS.día ${ }^{-1}$ ) para las distintas dietas.

\begin{tabular}{lcccc}
\hline dieta & $\overline{\mathrm{x}}(\mathrm{n}=4)$ & $\mathrm{DE}$ & $\mathrm{CV}(\%)$ & rango \\
\hline 1 & 2,74 & 0,49 & 17,7 & $2,14-3,17$ \\
2 & 2,82 & 0,32 & 11,8 & $2,55-3,26$ \\
3 & 2,76 & 0,49 & 17,6 & $2,09-3,24$ \\
4 & 2,64 & 0,30 & 11,6 & $2,24-2,98$ \\
\hline
\end{tabular}

$\overline{\mathrm{X}}$ promedio, DE: desvío estándar, $\mathrm{CV}$ : coeficiente de variación. Las diferencias entre promedios fueron no significativas.

Tabla 6. Conversión alimenticia (alimento/ganancia; $\mathrm{kg})$ para las distintas dietas.

\begin{tabular}{lcccc}
\hline dieta & $\overline{\mathrm{x}}(\mathrm{n}=4)$ & $\mathrm{DE}$ & $\mathrm{CV}(\%)$ & rango \\
\hline 1 & 2,73 & 0,61 & 22,5 & $2,00-3,49$ \\
2 & 2,64 & 0,38 & 14,3 & $2,11-2,99$ \\
3 & 2,88 & 0,64 & 22,1 & $1,96-3,38$ \\
4 & 2,54 & 0,31 & 12,1 & $2,22-2,98$ \\
\hline
\end{tabular}

$\overline{\mathrm{X}}$ promedio, DE: desvío estándar, CV: coeficiente de variación. Las diferencias entre promedios fueron no significativas.

En conclusión, surge que las variables productivas analizadas en este trabajo no variaron significativamente según las dietas ensayadas, por lo cual se induce que los cereales tradicionales como el maíz pueden ser parcialmente sustituidos sin inconvenientes por harina integral de mandioca en la alimentación de cerdos en crecimiento.

Agradecimientos: Al MV, Pedro A. Zeinsteger (FCV-UNLP), MV Pablo A. Rivas (ERAGIA) y al MV José A. González (Servicios Veterinarios Profesionales). 


\section{REFERENCIAS}

1. Acurero GA, Alvarado LR, Pérez SA, Álvarez RG, Cuaimara MR. 1981. La harina de batata (Ipomoea batata) como fuente energética en raciones para cerdos en crecimiento. Rev Ciencias Vet 10: 1407-1414.

2. Acurero GA, Alvarado LR, Álvarez RG, Pérez SJ, Capó E, Garbati ST. 1993. Efectos bioeconómicos de la sustitución parcial de los cereales por harina de batata en raciones para cerdos en crecimiento. Zoot Trop 11: 117128.

3. Alvarado LR, Álvarez RG, Acurero GA. 1977. Utilización de la harina de batata (Ipomea batata) en la alimentación del cerdo. Rev Cs Vet (Maracay, Venezuela) 6: 955.

4. Álvarez GR, Alvarado LR. 1975. Yuca como fuente energética en la alimentación de los cerdos. Boletín Técnico $M A C$ (Venezuela) 2: 77-83.

5. Angulo M, Lopez W, Brinke HW. 1979. Yuca, camote y grano de soya en engorde de cerdos. Memorias Asoc Latin Prod Anim 14: 64.

6. Belmar-Caso R. 1998. Recursos no convencionales en la alimentación de animales no rumiantes. Informe Departamento Nutrición Animal FMVZ, Universidad Autónoma de Yucatán (México), 10 p.

7. Bolhuis GG. 1954. The toxicity of cassava roots. Neth $J$ Agr Sci 2: 176-185.

8. Buitrago JA. 1990. La yuca en la alimentación animal, Ed. Centro Internacional de Agricultura Tropical (CIAT), Cali, Colombia, $446 \mathrm{p}$.

9. Campabadal CM. 1985. Utilización de la mandioca en la alimentación porcina. Boletín Asociación Americana de Soya (México) 85: 14.

10. Campabadal, CM. 1986. Utilización de subproductos agroindustriales en la alimentación de cerdos. Boletín Asociación Americana de Soya (México) 71: 12.

11. Chicco CF, Garbati ST, Muller-Haye B, Vecchinacce H. 1972. La harina de yuca en el engorde de cerdos. Rev Agron Trop 22: 599-603.

12. CIAT, Centro Internacional de Agricultura Tropical. 2002. La yuca en el tercer milenio. Sistemas modernos de producción, procesamiento, utilización y comercialización. Publicación CIAT 327, Cali (Colombia), p. 3.

13. FAO. 2006. Anuario. Disponible en: http://www.fao.org.

14. González C, Vecchionacce H, Díaz I, Ortiz V. 1997. Utilización de harina cruda de raíz de mandioca (Manihot esculenta c.) y harina cruda de cormos de ocumo chino (Colocasia esculenta c.) en la alimentación de cerdos. Arch Lat Prod Anim 5: 277-279.

15. González C, Díaz I, Vecchionacce H. 1999. Uso de raíces y tubérculos en la alimentación de cerdos $V$ Encuentro sobre nutrición y producción de animales monogástricos, Maracay (Venezuela), p. 17.

16. Jaramillo L, Herrera H. 1970. Rendimiento de variedades de yuca. Anales VIII Reunión de Filotecnia, Bogotá (Colombia), p. 22-28.

17. Johnson RM, Raymond WE. 1965 . The chemical composition of some tropical food plants. Trop Sci 7: 109-115.
18. Koo NW, Kim DK. 1974. The feeding value of dried sweet potato pulp for growing-finishing swine. Res Rural Develop (S. Korea) 16: 97-101.

19. Ly J. 1993. Fisiología digestiva del cerdo. Anales II Curso sobre biometría, fisiología, nutrición y alimentación de cerdos, Maracay (Venezuela), p. 125.

20. Maust LE, Scott ML, Pond WG. 1972. The metabolizable energy of rice bran, cassava flour and blackege cowpeas for growing chickens. J Anim Sci 35: 953-957.

21. Montaldo A. 1973. Importancia de la mandioca en el mundo actual con especial referencia a Venezuela. Rev Fac Agron UCV 22: 41-44.

22. Montilla JJ. 1973. Uso de la mandioca en la alimentación de aves, cerdos y vacunos. Rev Fac Agron UCV 22 :115125 .

23. Morel F. 1998. Cultivo de la Mandioca. Boletín INTAEEA Cerro Azul (Misiones, Argentina) No 24, 17 p.

24. Nartey F. 1968. Studies on Cassava manihot utilissima, Pohl. 1. Cyanogenesis : the biosynthesis of linomarina and lotaustralin in etiolated seedlings. Phytochemistry 7 : 1307-1312.

25. Natural Resources Conservation, NRC. 1998. Nutrient requirements of swine, 10th Ed., National Academy Press, Washington, $614 \mathrm{p}$.

26. Parra N. 1991. Utilización del follaje de mandioca como fuente proteica en el engorde de cerdos. Boletín Soc Vet Venez Esp Cerdos 2: 38.

27. Pochon DO, Navamuel JM, Koslowski HA, Balbuena O, Rivas PA. 2005. Aceptabilidad y selección de mandioca (Manihot sculenta Crantz) como sustituto de maíz en raciones para cerdos en crecimiento. http://www.unne.edu. ar/Web/cyt/com 2005/4-Veterinaria/V-045.pdf.

28. Pond WG, Maner JH. 1984. Swine production and nutrition, AVI Publishing Co, Wesport, Connecticut, $89 \mathrm{p}$.

29. SAGPyA, Secretaría de Agricultura, Ganadería, Pesca y Afines. 2001. Análisis FODA del sector porcino nacional. www.sagpya.mecon.gov.ar/0-1/porcinos.

30. Savón L, Gutiérrez O, Martínez L, González T, Orta M. 1999. Manual teórico práctico de productos fibrosos, Ed. EDICA, La Habana (Cuba), 33 p.

31. Savón L, Scull I. 2001.Valor potencial de alimentos fibrosos tropicales para especies monogástricas. Anales VI Encuentro Regional de Nutrición y Alimentación de Animales Monogástricos, La Habana (Cuba) p. 36.

32. Sgroppo SC, Fusco AJ, Avanza JR. 1986. Secado de mandioca. Anales II sesiones de comunicaciones sobre investigación y desarrollo en disciplinas relacionadas con la química. FACENA, UNNE (Corrientes, Argentina), p. $20-21$.

33. Sinisterra AA. 2002. Efectos en el rendimiento productivo de cerdos en la etapa de acabado por el reemplazo del total de maíz por harina de yuca en la dieta. Trabajo de grado, Universidad de San Buenaventura, Cali (Colombia), $\mathrm{p}$. $38-40$.

34. Spiegel Murray R. 1991. Estadística, McGraw-Hill, Madrid, $345 \mathrm{p}$.

35. Uset OA. 2009. Utilización de raíces y parte aérea de mandioca en la alimentación animal. Boletín Técnico EEA INTA Montecarlo (Misiones, Argentina) 62: 6-11. 